\title{
Caracterización de la enseñanza y el aprendizaje de la educación en la Escuela de Aviación del Ejército ${ }^{1}$
}

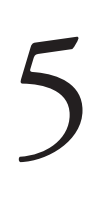

https://doi.org/10.21830.9789585350663.05

\author{
Edgar Giovanny Ortiz Medina \\ Escuela de Aviación del Ejército
}

Resumen. La educación militar está ligada a la evolución de los ejércitos y es impartida por docentes que transmiten los conceptos doctrinales como parte del proceso de formación de las tropas. El capítulo analiza mediante un estudio de caso el uso de componentes didácticos y de evaluación que los docentes militares de la Escuela de Aviación del Ejército (EsAVE) desarrollan en el proceso de enseñanza-aprendizaje de la doctrina castrense, así como el impacto y las consecuencias de su aplicación dentro de la asimilación cognitiva del estudiante, que con el tiempo aplicará la teoría militar para la ejecución efectiva de operaciones. Se plantea una metodología mixta, de tipo exploratorio, transeccional, de carácter no experimental, que guarda relación con los resultados de la investigación. Los resultados comprueban la hipótesis inicial referente a la relación directa entre la aplicación de la didáctica por parte de los profesores y su efecto en el rendimiento académico de los estudiantes.

Palabras clave: educación militar; cognición; componentes didácticos; enseñanza; método de evaluación; operación militar.

1 Este capítulo presenta los resultados del proyecto de investigación "Estrategias didácticas basadas en la inteligencia emocional como herramienta para la formación del carácter en estudiantes”, del grupo de investigación en Aviación Militar de la Escuela de Aviación del Ejército (EsAvE), categorizado en C por Minciencias y con código de registro COL0077618. Los puntos de vista pertenecen al autor y no reflejan necesariamente los de las instituciones participantes.

2 Teniente Coronel del Ejército Nacional de Colombia. Profesional en Ciencias Militares de la Escuela Militar de Cadetes “General José María Córdova”. Especialista en Docencia Universitaria del Centro de Educación Militar. Especialista en Seguridad y Defensa Nacionales de la Escuela Superior de Guerra "General Rafael Reyes Prieto". Magíster en Educación de la Universidad Militar Nueva Granada. OrCID: https://orcid.org/0000-0002-0755-9443 - Contacto: edgar.ortizme@buzonejercito.mil.co 


\section{Introducción}

La investigación pretende someter a prueba la siguiente hipótesis: los componentes didácticos y métodos de evaluación que implementan los oficiales del Ejército Nacional de Colombia en el desempeño de su rol como profesores militares ayudan a desarrollar el proceso de enseñanza de la educación y la doctrina militar, con lo cual se incentiva la cognición del estudiante y se logra la base teórica inicial para la ejecución de operaciones militares. Este planteamiento focaliza al proceso cognitivo del estudiante, mediante la aplicación de la didáctica y la evaluación por parte del docente, lo cual demuestra de manera tangible que el uso de estos métodos incrementa la efectividad del proceso académico descrito.

El desarrollo de este propósito plantea un estudio de caso que determina una muestra representativa de los oficiales docentes militares, mediante la selección de un grupo de pilotos instructores de ala fija, orgánicos de la Aviación del Ejército, en el marco de un curso de Transición de Vuelo, con estudiantes miembros de la oficialidad que aspiran calificarse como copilotos en las diferentes aeronaves con las que cuenta la institución.

El inicio de la investigación parte de la premisa histórica de la enseñanza doctrinal de los profesores militares, la cual determina dos pilares fundamentales: la doctrina base fundamental de los ejércitos y el estudiante, como actor principal del proceso de cognición. Así pues, "concebir al educando como una ser activo, protagonista, reflexivo, producto de variadas interrelaciones sociales que ocurren en un contexto histórico-cultural específico y que reconstruye el conocimiento con los demás" (Chaves, 2011, p. 64).

En este sentido, es fundamental determinar el grado de preparación pedagógica que tienen los gestores del conocimiento militar y conocer si su designación es o no producto de hechos circunstanciales sin fundamentaciones didácticas ni conocimiento de los métodos de evaluación. Respecto a este planteamiento, Méndez (2013) afirma:

A las escuelas y centros de educación militar llegan profesores (oficiales, suboficiales, civiles), cuya función principal se limita a transmitir, enseñar o explicar sus conocimientos sobre un tema específico; en términos generales repiten las mismas informaciones recibidas en su proceso formativo, sin tomar 
conciencia de que puede conseguirse con estrategias y escenarios mucho más atractivos, interesantes y útiles. (p. 8)

Este capítulo expone de forma pertinente, lógica y coherente el proceso investigativo que se llevó a cabo durante más de dos años de trabajo de campo recopilando datos históricos de cinco años en retrospectiva, el cual refleja la "coherencia de la formación integral del militar profesional que responde a los retos del actual mundo globalizado" (Terreros, 2012, p 9).

Así pues, se presentan cuatro fases en las que se desarrolla y comprueba la hipótesis en su totalidad: (1) Contrastación entre objetivos y marco teórico, (2) Diseño de instrumentos, (3) Recolección de datos y (4) Procesamiento y análisis de la información.

Como aporte final a los planteamientos teóricos y prácticos de la investigación, se presentan los resultados y las conclusiones, los cuales coadyuvan de manera directa a la educación militar, brindan herramientas de valor para el rol del profesor militar e incentivan la cognición del estudiante para la aplicación de la doctrina aprendida en el desarrollo de operaciones militares. Este aporte de la investigación se sustenta en que "el docente es el responsable profesional del trabajo en el aula [y por tanto] le corresponde elaborar una propuesta de programa en el que fundamentalmente defina las estrategias de enseñanza” (Díaz, 1997, p. 66).

Para finalizar, es importante tener en cuenta el concepto que la institución tiene del título de profesor militar, que es una distinción académica representativa destinada a incentivar la labor docente de los oficiales y suboficiales de las Fuerzas Militares que se desempeñen como profesores militares en institutos de educación castrense.

Así pues, de acuerdo con la normatividad vigente, existen cinco categorías, cada una con requisitos específicos, como registro de horas mínimas de clases, verificación de Curso Básico de Instructores y permanencia en instituciones o escuelas de formación militar, entre otros. Inicialmente, en orden de categorías se otorga la quinta, cuarta y tercera, que la emite el comandante de la respectiva Fuerza. Posterior a estas continúan la segunda y la primera, que las otorga el Comandante General de las Fuerzas Militares, de manera que son las máximas categorías del título, de acuerdo con lo previsto en los decretos 1495 de 2002 y Decreto 1070 de 2015, respectivamente. 


\section{Marco teórico}

El desarrollo de este trabajo está basado en autores y teorías que soportan el peso de la investigación para comprobar la hipótesis planteada. Como temáticas centrales del proceso se muestran dos variables fundamentales: los oficiales pilotos de ala fija del Ejército en el rol de instructor-estudiante y el uso de los componentes didácticos y métodos de evaluación (Rodríguez, 1999).

Inicialmente, los profesores militares y los estudiantes fueron analizados desde la corriente del constructivismo, con autores destacados como Lev Vygotsky, por cuanto postula la creación de conocimiento a partir del estudio de las personas en interacción social, que para el caso de estudio se presenta como la interacción y relación entre los diferentes actores presentes en el proceso académico, tales como el profesor militar y sus estudiantes, los estudiantes entre sí y los dos grupos en sinergia con el entorno circundante (Duque \& Packer, 2014, p. 52). Asimismo, se recurre al trabajo de Alexander Kapp para analizar las corrientes y el desarrollo del concepto de andragogía como la enseñanza de los adultos (Alonso, 2012, p. 18). Finalmente, se toman las bases modernas del concepto andragógico actual de Malcolm Knowles (Mena et al., 2014).

Los componentes didácticos y los métodos de evaluación se soportan en las teorías de Juan Amos Comenio, con su obra la Didáctica magna, y en el trabajo de Ángel Díaz Barriga y su hermana Frida, quienes constituyen un referente sine qua non en el desarrollo del concepto en Latinoamérica, con una mirada más actual: "La didáctica es el foco intelectual y organizativo de los procesos educativos en los centros de enseñanza” (Díaz, 1998, p. 103).

Respecto a la revisión de modelos de evaluación, se toma como referencia a Ralph Tyler (Pérez, 2014), padre de la orientación de objetivos, quien hace aportes desde el método evaluativo a la práctica docente para alcanzar el método de evaluación general (López, 2005, p. 40).

Finalmente, un referente importante en este trabajo es David Ausubel, quien plantea la Teoría del Aprendizaje Significativo, según la cual la estructura de los conocimientos previos condiciona los nuevos conocimientos y experiencias, y estos, a su vez, modifican y reestructuran los existentes (Álzate, 2015, p. 5). Por supuesto, los estudiantes inmersos en la transición de vuelo como parte de la doctrina militar no son ajenos a este planteamiento y se constituyen como un ejemplo concreto de la representación teórica que refuerza la hipótesis. 


\section{Métodos}

La presente investigación es de tipo mixto, de manera que utiliza fuentes de datos cuantitativos y cualitativos, que luego de ser descritos permiten la interpretación del investigador (Hernández et al., 2014).

En correspondencia, se desarrollaron dos etapas generales de la investigación: en primer lugar, en la cuantitativa se recolectó información mediante la constitución de una base de datos, la cual permitió caracterizar la población en función de las variables implícitas en la investigación, relacionadas con el diseño del método de evaluación y la didáctica, que "describe la enseñanza cuando da cuenta de sus partes constitutivas y de sus cualidades" (Picco \& Orienti, 2017, p. 12). En segundo lugar, en la etapa cualitativa se realizaron entrevistas semiestructuradas con base en los análisis de la caracterización.

\section{Nivel y tipo de la investigación}

Se considera de tipo no experimental, exploratoria en cuanto se basa en la observación de un fenómeno tal y como se presenta de forma natural para posteriormente analizarlo (Hernández et al., 2014, p. 85). Asimismo, se discriminan los datos a través de correlaciones de variables dependientes e independientes.

\section{Presentación del caso}

La investigación se llevó a cabo en la ciudad de Bogotá, Colombia, en la Escuela de Aviación del Ejército Nacional (EsAve), ubicada en el Aeropuerto El Dorado, en el campo aéreo José Joaquín Matallana Bermúdez, en donde también se ubica el Batallón de Aviación N. ${ }^{\circ} 1$ "Aviones" (BAAvi), unidad táctica de la cual dependen operacionalmente las aeronaves de ala fija de la Aviación del Ejército Nacional de Colombia.

El periodo de análisis de la investigación abarca del 2015 al 2019. En el último año, el BAAVi poseía una flota total de 25 aeronaves de diferentes tipos, entre las cuales se cuentan Cessna, Beechcraft, Casa, Twin Commander y Antonov.

Los individuos objeto de estudio son pilotos instructores y estudiantes orgánicos del BAAVI y se constituyen como la muestra representativa de la institución en docentes y estudiantes. Este grupo heterogéneo cuenta con dife- 
rentes tiempos de servicio dentro del Ejército y variación en el número de horas de vuelo como pilotos instructores. Los estudiantes cuentan con doscientas horas de vuelo al iniciar el curso de Transición.

Durante estas horas de vuelo como pilotos de aviones, todos los oficiales cumplen la misión institucional de la unidad táctica, descrita a continuación como lo enuncia el Manual de empleo táctico del Batallón de Aviones del Ejército Nacional (2012a):

Cumple misiones de combate, apoyo de combate, apoyo y servicios para el combate, con el propósito de sostener el poder operacional de las unidades de ala rotatoria de la aviación de Ejército y ejecutar misiones especiales y de inteligencia técnica, con el fin de incrementar la sinergia operacional. (p. 19)

\section{Población}

La población objeto de estudio se segmentó en dos grupos: (1) oficiales pilotos instructores de ala fija y (2) estudiantes en transición.

Para caracterizar la población de estudio, se empleó la definición de soldado aviador y piloto consignada en el Manual de misiones tácticas de aviación (Ejército Nacional, 2012b) y el Reglamento de vuelo (Ejército Nacional, 2013):

Se consideran soldados aviadores todo el personal militar o civil que labora para el Ejército Nacional de Colombia y que se encuentra asignado a una Unidad de Aviación. Una tripulación se define como el conjunto de personas debidamente instruidas, entrenadas y calificadas, dedicadas a la maniobra y operación de una aeronave para el cumplimiento de una misión de vuelo. [...]. Los pilotos son los individuos instruidos entrenados y calificados que forman parte de una tripulación y que comandan las aeronaves desde las estaciones de vuelo con acceso a los controles, siendo los responsables de una operación segura y eficaz. (p. 23)

En este entendido, se explica a fondo cada grupo poblacional entre instructores de vuelo y estudiantes al inicio del curso de vuelo dentro de la Aviación del Ejército Nacional.

\section{Oficiales instructores de vuelo de ala fija}

Inicialmente, para el estudio se identificó que el total de instructores de ala fija de la Aviación del Ejército es de veintidós instructores. No obstante, se 
delimitó la población de estudio porque siete pilotos son civiles contratados y no son relevantes para el proceso investigativo, por tanto, se inició la investigación con quince oficiales instructores de vuelo.

Esta población fue seleccionada debido a su proceso vivencial, que comprende desde su inicio como copilotos hasta su cargo como piloto instructor. Se trata de personal que ha sido capacitado siguiendo todos los pasos del aprendizaje en la institución y en concordancia con las normas doctrinales vigentes, como lo enuncia el Manual de misiones tácticas de aviación del Ejército Nacional (2012b):

La doctrina de aviación del Ejército se enfoca en la integración y sincronización de fuerzas de la aviación dentro de los conceptos y doctrina operacional del comandante de los componentes de tierra. [...]. Las unidades de aviación operan con las unidades de tierra como un miembro totalmente integrado del equipo de armas combinadas, las unidades de la aviación desarrollan misiones de combate, apoyo de combate y apoyo y servicios para el combate. (p. 34)

Su función es instruir a todos los pilotos que cíclicamente pasan por las aulas de la Escuela de Aviación del Ejército, con el objetivo de cumplir una capacitación llamada Transición de Vuelo, consistente en aprender a volar un avión específico dentro de los equipos de vuelo del Batallón. Este proceso se rige por la siguiente premisa: "La relación 3.0, uno (1) por cada (5) pilotos y un total general de nueve (9) por cada unidad táctica de aviación" (Ejército Nacional, 2017a).

\section{Estudiantes nuevos para transición de equipo}

Para precisar el uso del término estudiante nuevo en el contexto de la enseñanza de vuelo, es necesario aclarar que estos estudiantes han pasado un proceso de formación como pilotos básicos en diferentes academias de pilotaje, por tanto no son estudiantes que se enfrentan por primera vez al vuelo de una aeronave, sino que cuentan con experiencia mínima de doscientas horas de vuelo.

Para llegar a este punto deben someterse a una selección rigurosa y excluyente en la que se miden las capacidades especiales de los oficiales, los conocimientos teóricos clasificados mediante exámenes y test, y la verificación del estado de salud física y mental que posee cada candidato (Ejército Nacional, 2015): 
Con el fin de comprender la selección de estudiantes de vuelo dentro del cuerpo de oficiales del Ejército Nacional de Colombia, se contextualizará el proceso que sigue un aspirante desde su ingreso a la formación en el alma mater de la Fuerza, hasta la selección dentro de un curso de vuelo. (p. 45)

\section{Contenido curricular del curso de vuelo}

El curso de Transición a los diferentes equipos de vuelo de ala fija en el BAAVI está constituido por dos fases: la fase de tierra y la fase de vuelo, con una duración total de cinco semanas, cuyo componente curricular se explica a continuación (Ejército Nacional, 2017b).

\section{Fase de tierra}

Las tres primeras semanas del curso para "pilotos nuevos" inician en el aula de clases, donde se desarrolla el plan curricular formal con el siguiente temario definido en el reglamento aeronáutico de Colombia (Unidad Administrativa Especial de Aeronáutica Civil [UAEAC], 2020, p. 58):

- Generalidades de la aeronave.

- Sistema eléctrico.

- Sistema de combustible.

- Sistema hidráulico.

- Sistema neumático.

- Sistema del motor.

- Sistema neumático.

- Sistema de frenos.

- Tren de aterrizaje.

- Otros sistemas complementarios.

- Visualización externa o prevuelo.

- Límites y emergencias.

A través de estos temas el instructor de vuelo enseña, mediante diferentes técnicas, los componentes teóricos del funcionamiento, la aplicación y el uso de los diferentes sistemas que posee la aeronave, con el fin de que el estudiante identifique de manera clara dichos componentes necesarios para desarrollar un vuelo. 
Para desarrollar las temáticas listadas, el instructor de vuelo utiliza la guía Pilot Operating Handbook ( $\mathrm{PoH}$ ) o manual de vuelo de la aeronave, que contiene los datos específicos sobre los sistemas de la aeronave, sus límites y emergencias, así como las listas de chequeo, que son procedimientos que el piloto realiza de forma secuencial en el desarrollo de un vuelo.

La evaluación de estas temáticas se realiza de forma escrita mediante exámenes de conocimiento, en los cuales el estudiante debe alcanzar un mínimo de $80 \%$ de respuestas correctas para aprobarlos, y exámenes de límites y emergencias, en los cuales este porcentaje debe ser del $100 \%$.

A partir del curso de la fase de tierra de la aeronave, el estudiante aprobará o reprobará con los valores descritos y continuará a la fase del vuelo, donde aplicará los conceptos teóricos aprendidos.

\section{Fase de vuelo}

La formación en esta fase tiene una duración de dos semanas. Como su nombre lo indica, se realiza en la labor del vuelo propiamente dicha, dentro de una cabina de aeronave. En esta fase se busca que el piloto ponga en práctica la operación de los conceptos teóricos desarrollados en clase para medir su habilidad en las diferentes misiones que describe el reglamento aeronáutico de Colombia (UAEAC, 2020, p. 62). Algunas de estas funciones son:

- Familiarización, maniobras y control de vuelo.

- Trabajo de pista (despegues y aterrizajes).

- Límites y emergencias en vuelo.

- Vuelo por instrumentos.

- Chequeo final (unión de las anteriores fases).

En esta fase el instructor de vuelo utiliza el Pilot Training Manual (Ртм), que es el manual de entrenamiento del piloto. En este documento se compilan las maniobras, los procedimientos y demás información requerida para aprobar la formación en esta fase. Además, se utiliza la aeronave como medio de enseñanza, con el método de aprender haciendo.

Al finalizar cada misión y la fase en general, el instructor evalúa con letras el desempeño del estudiante determinando si el desempeño del piloto alumno fue Satisfactorio (S) o No Satisfactorio (N). Este resultado es excluyente dentro 
del proceso, ya que si la evaluación es no satisfactoria, el oficial no se puede graduar de piloto y por consiguiente no podrá ejercer misiones de vuelo.

\section{Fases del proceso de trabajo de campo}

El proceso investigativo se llevó a cabo en cuatro fases, en las cuales se logró compilar todo el desarrollo de la pregunta problema. Mediante la aplicación de estas fases se organizó el trabajo con líneas de tiempo definidas y se aportó de manera constante a la recolección y verificación de la información, así como al soporte teórico y metodológico de la investigación, para cumplir los objetivos y dar solución al problema planteado, que supone una mejora en el proceso estudiantil de los alumnos (Sotelo \& Arvelo, 2016).

\section{Fase 1. Contrastación entre los objetivos y el marco teórico}

Dentro del desarrollo metodológico y conceptual del problema de investigación aclaramos e identificamos diversos núcleos temáticos, como la experiencia de los pilotos instructores, los diferentes tipos de equipo de vuelo, las horas de vuelo totales de cada instructor, así como las horas en su ejercicio como docente, entre otros.

Esta información permitió establecer las categorías y variables de análisis, como las herramientas didácticas en clase, la metodología, la evaluación y la experiencia en horas de vuelo, que son aplicables al análisis del rol instructor docente militar piloto para caracterizar tanto el proceso de enseñanza-aprendizaje como la población de instructores y alumnos, de acuerdo con los lineamientos del Proyecto Educativo de las Fuerzas Armadas (PefA) (Ministerio de Defensa, 2008a).

Con lo anterior se logró contrastar los objetivos propuestos con el marco teórico, que es coherente y pertinente, lo cual que se refleja en el desarrollo de todo el proceso investigativo.

\section{Fase 2. Diseño de instrumentos}

En esta fase se diseñaron tres instrumentos para recolectar los datos: en primer lugar, se constituyó una matriz para la construcción de una base de datos que permitiera caracterizar la población. En segundo lugar, se elaboró un cuestionario escrito para hacer un sondeo sobre las herramientas didácticas y los 
métodos de evaluación que implementan los instructores. Finalmente, en tercer lugar, se diseñó un cuestionario de entrevista semiestructurada para caracterizar dichas herramientas en función del análisis de la relación entre los instructores y los resultados de aprobación y reprobación de los pilotos-alumnos, siguiendo el parámetro del Plan Estratégico del Sistema Educativo (PESE) (Ministerio de Defensa, 2008b).

El sondeo contó con tres secciones de preguntas: (1) información general sobre el proceso de enseñanza-aprendizaje, (2) identificación de las herramientas didácticas implementadas en el proceso de formación e (3) identificación de las herramientas de evaluación implementadas en el aula. El objetivo de este instrumento fue recolectar información de carácter descriptivo para identificar las herramientas y establecer la base de indagación que se desarrollaría en las entrevistas.

Para la fase de análisis cualitativo se diseñaron entrevistas semiestructuradas por medio de un cuestionario, con el cual se indagó por las características específicas, los contextos de utilización y los objetivos pedagógicos que persigue el instructor con la implementación de las herramientas inventariadas. Este instrumento se diseñó luego de obtener los resultados del sondeo de las herramientas, con el que también se obtuvo información sobre los porcentajes de uso y no uso de dichas herramientas.

El cuestionario de entrevista contó con un total de veinte preguntas, a través de las cuales se indagó sobre las herramientas inventariadas, luego de ser seleccionas aquellas con mayor porcentaje de uso. El promedio de duración de las entrevistas fue de una hora, siempre siguiendo los lineamientos curriculares para las Fuerzas Militares definidos en el SEFA (Ministerio de Defensa, 2010).

\section{Fase 3. Recolección de datos}

La recolección de datos se realizó en el auditorio de la Esave. Primero se reunió a la totalidad de los instructores con el fin de dar las orientaciones generales para el diligenciamiento del cuestionario. Posteriormente, cada uno de los participantes desarrolló el cuestionario sin limitación de tiempo. Al momento de la aplicación del instrumento se hizo énfasis en la necesidad de que la información suministrada fuera precisa y diera cuenta de las prácticas reales que desarrollaba cada uno de los instructores en su práctica docente. 
Luego de esta aplicación se realizaron las entrevistas a aquellos instructores que en el análisis de la base de datos se identificaron con la mayor tasa de aprobación y de reprobación de los estudiantes en los exámenes de la fase de aire y de tierra. Específicamente, se seleccionaron los cinco instructores con mayor tasa de aprobación y los cinco con mayor de reprobación.

Con los resultados obtenidos del sondeo se identificaron las herramientas didácticas y de evaluación que implementan los instructores y se determinó el porcentaje de uso y no uso en una diferencia porcentual del $46,7 \%$ y el parámetro de no uso vs uso, en una diferencia porcentual del $20 \%$, en el total de variables de análisis. Con base en esta discriminación por variable se seleccionaron las herramientas sobre las cuales se solicitaría la descripción de uso a la población de estudio.

\section{Fase 4. Procesamiento y análisis de la información}

A partir de la fase inicial de recolección se constituyó una base de datos de caracterización de la población con base en la información entregada por el BAAVi. Específicamente, se compiló información en variables de análisis iniciales tales como número de caso, horas de vuelo totales, horas de vuelo como instructor, año de desarrollo de la instrucción, cursos dictados, número de estudiantes, resultados fase de tierra, resultados fase de vuelo, dato de aprobaciones o reprobaciones por cada registro. Como resultado se obtuvo una matriz de datos sobre la cual se realizó un análisis de frecuencias y porcentual.

De esta manera, se organizaron tablas de frecuencia por instructores y alumnos sobre los siguientes aspectos: equipo de vuelo, horas totales como instructor, tiempo en ańos como instructor, número de estudiantes, evaluaciones en las fases de tierra y vuelo, tasa de aprobación y reprobación. Después se realizaron cruces de información de forma progresiva y se verificó las posibilidades de cruces de información necesaria referente a instructores y a estudiantes, teniendo en cuenta la totalidad de los registros.

Esta base de datos permitió organizar la información producida por el BAAVI en el periodo 2015-2019 sobre la población de estudio. Uno de los principales análisis elaborados sobre estos datos fue la descripción del desempeño de cada instructor en los cursos de vuelo impartidos, en relación con el resultado general de aprobación o reprobación de los estudiantes a los cuales se instruyó en ese lapso. 
Este resultado se obtuvo a partir de las variaciones porcentuales y de frecuencia de aprobación y reprobación de cada instructor. Es decir, de la relación directa entre la variable: cantidad de estudiantes por instructor y porcentaje de aprobaciones y reprobaciones durante el periodo 2015-2019.

También se llevó a cabo un análisis porcentual y de frecuencia sobre el desempeño estudiantil de los pilotos estudiantes. Diferentes variables de discriminación poblacional, especialmente las horas de vuelo, y los porcentajes de aprobación y reprobación de los exámenes de tierra y aire permitieron segmentar la población estudiantil.

De forma concomitante, se analizaron los datos obtenidos tras el sondeo, en el cual se identificaron las herramientas didácticas y de evaluación enunciadas por los pilotos-instructores como aquellas implementadas en el aula de clase para la enseñanza de las materias propias del curso de vuelo para oficiales alumnos en proceso de formación como pilotos del Ejército Nacional de Colombia.

El resultado del sondeo dio cuenta de tres categorías de análisis sobre las que se agruparon las herramientas inventariadas: (1) las herramientas didácticas para la clase, (2) la metodología que se desarrolla en clase y su relación con el uso de las herramientas, y (3) las herramientas de evaluación utilizadas por el instructor durante el curso.

Con base en estas categorías se agruparon las diversas herramientas y se elaboró un análisis de frecuencias por el total de instructores. Asimismo, con las tablas de frecuencia se estableció un análisis ponderado de las diferentes herramientas que fueron identificadas. Finalmente, con esta información se realizó un análisis del uso y no uso de las herramientas por el total de instructores.

A partir de los resultados iniciales del sondeo se seleccionaron las herramientas didácticas y de evaluación por los resultados ponderados de las variables uso y no uso, con un porcentaje de uso del 73,3 \% y de no uso del 23,3 \%. Sobre esta base se creó el segundo instrumento de recolección de información de tipo cualitativo: la entrevista semiestructurada.

Este instrumento se aplicó a dos subgrupos de la población de estudio: el subgrupo 1, conformado por los instructores con mayor tasa de aprobación, y el subgrupo 2, que corresponde a los instructores con mayor tasa de reprobación. 
El principal objetivo de la entrevista fue obtener la información necesaria para caracterizar de manera más detallada las herramientas didácticas y de evaluación identificadas, así como establecer una posible relación entre el uso y no uso de estas herramientas con los resultados de aprobación y reprobación por instructor. $\mathrm{Al}$ respecto, cabe señalar que se recordó siempre a los docentes que "para desempeñar la labor de maestro resulta indispensable disponer de materiales didácticos y definir los procedimientos de trabajo para evitar que el nivel de complejidad del aprendizaje sea mayor" (Andrade, 2011, p. 28).

Para procesar la información se transcribieron y codificaron las entrevistas, a partir de lo cual se hizo un análisis por herramientas utilizando una matriz. En la constitución de las categorías de análisis se emplearon las herramientas didácticas identificadas y seleccionadas. De esta forma, se elaboró un análisis categorial de las entrevistas por cada caso de estudio y finalmente se realizó un análisis por categoría.

\section{Resultados}

A continuación se exponen los resultados de los análisis que se hicieron a la información recolectada mediante tres instrumentos principales: (1) base de datos con la caracterización de la población de estudio (pilotos instructores y pilotos estudiantes), (2) sondeo sobre las herramientas didácticas y de evaluación implementadas en el aula por los pilotos instructores, y (3) entrevistas de caracterización de las herramientas inventariadas y seleccionadas.

\section{Tablas de frecuencia obtenidas con la base de datos}

En el análisis de las tablas de frecuencia se tuvo en cuenta que durante el periodo de análisis (2015-2019) todos los cursos de vuelo fueron cubiertos por quince instructores. Se recopiló la información sobre inicio, término, desempeño valorado en resultados de la fase de vuelo y fase de tierra, sobre el número inicial de 328 estudiantes que se encontraban inscritos en los diferentes cursos de vuelo.

Se generaron quince tablas de frecuencia en total, pero solo se presentan las cinco que compilan la información relevante para comprender la relación entre el desempeño de los pilotos-estudiantes y la implementación de las herramientas didácticas y los métodos de evaluación por parte de los profesores militares. 
Tabla 1. Total de estudiantes periodo 2015-2019

\begin{tabular}{cccc}
\hline Años históricos & Semestre I & Semestre II & Total \\
\hline 2015 & 49 & 12 & 61 \\
2016 & 21 & 38 & 59 \\
2017 & 20 & 33 & 53 \\
2018 & 56 & 26 & 82 \\
2019 & 73 & & 73 \\
\hline Total, general & 219 & 109 & 328 \\
\hline
\end{tabular}

Fuente: elaboración propia.

A lo largo del periodo 2015-2019, el número total de estudiantes que iniciaron el curso fue de 328. Asimismo, se evidencia que el año 2015 tuvo la menor cantidad, con 61 estudiantes. Por su parte, el año 2018 demostró la máxima cantidad, con un total de 82. El promedio anual está en 65 estudiantes (tabla 1).

Tabla 2. Equipos de vuelo por estudiantes al inicio del curso de vuelo

\begin{tabular}{cc}
\hline Equipo de vuelo & Estudiantes \\
\hline AC-90 & 22 \\
Antonov & 33 \\
Beechcraft & 68 \\
C-208B & 117 \\
C-212 & 38 \\
Simulador de vuelo & 50 \\
\hline Total, general & 328 \\
\hline
\end{tabular}

Fuente: elaboración propia.

Se evidencia que de los 328 registros expresados en estudiantes que iniciaron proceso de vuelo, 117 recibieron curso de vuelo en el equipo C208 B Grand Caravan, mientras 22 estudiantes lo desarrollaron en el equipo AC-90 Turbo Commander. Asimismo, 68 de ellos iniciaron capacitación en Beechcraft y 33 estudiantes se capacitaron en el equipo Antonov 32. El resto de los estudiantes iniciaron capacitación en C-212 y en simulador de vuelo, sumando un total de 88 . Se infiere que la rotación de estudiantes en el equipo 
C-208B es mayor que en cualquier otro equipo de Batallón, por lo cual es necesario que la cantidad de instructores sea mayor en proporción a los otros equipos (tabla 2).

Tabla 3. Tiempo como piloto instructor en meses y cantidad de asignados

\begin{tabular}{lc}
\hline Meses como piloto instructor & Estudiantes \\
\hline a) 24 meses o menos & 37 \\
b) Mayor a 24 o menor a 48 meses & 69 \\
c) Mayor a 48 o menor a 96 meses & 162 \\
d) Mayor a 96 meses & 60 \\
\hline Total, general & 328 \\
\hline
\end{tabular}

Fuente: elaboración propia.

De los 328 estudiantes totales, 162 recibieron el curso por parte de un instructor que llevaba entre 48 meses y 96 meses en su cargo, mientras que 37 estudiantes fueron capacitados por un docente que tenía entre 1 y 24 meses. Asimismo, 60 estudiantes iniciaron curso de vuelo con un instructor con más de 96 meses en su función (tabla 3). Por tanto, cuantas menos horas tenga en la función como piloto instructor, menor será el número de estudiantes a su cargo. Lo anterior tiene como propósito incrementar la seguridad aérea en las operaciones aeronáuticas, pues a mayor tiempo de vuelo aumenta también la responsabilidad en el desarrollo de la labor docente y por ello exige al mismo tiempo mayor dominio de métodos, estrategias y prácticas didácticas fundamentadas en el constructivismo.

Tabla 4. Horas de vuelo como piloto instructor en meses y cantidad de estudiantes

\begin{tabular}{lc}
\hline Horas de vuelo como piloto instructor & Estudiantes \\
\hline a) Menos de 1.000 horas & 37 \\
b) Entre 1.000 y 2.500 horas & 155 \\
c) Entre 2.501 y 5.000 horas & 119 \\
d) Más de 5.000 horas & 17 \\
\hline Total, general & 328 \\
\hline
\end{tabular}

Fuente: elaboración propia. 
De los 328 registros expresados en estudiantes que iniciaron curso de vuelo, 155 recibieron el curso por parte de un instructor que ostentaba entre 1.000 y 2.500 horas de vuelo (tabla 4), lo que nos lleva a pensar que por regla general un piloto instructor con bastantes horas de vuelo tendrá la experiencia y capacidad de entrenar mayor cantidad de estudiantes de forma segura y con calidad. De manera contraria, la máxima experiencia en horas de vuelo no siempre representa un indicador de volumen de estudiantes, ya que se pudo comprobar que los pilotos instructores con más de 5.000 horas de vuelo dentro del Ejército Nacional entrenan poca cantidad de pilotos en los equipos por el desempeño de cargos de liderazgo y diferentes responsabilidades.

Tabla 5. Total de aprobaciones y reprobaciones en los exámenes de tierra y aire

\begin{tabular}{ccc}
\hline Total, aprobación y reprobación de exámenes & Estudiantes & Porcentaje \\
\hline Aprobado & 242 & $73,8 \%$ \\
Reprobado & 86 & $26,2 \%$ \\
\hline Total, general & 328 & $100 \%$ \\
\hline
\end{tabular}

Fuente: elaboración propia.

Se puede observar que del total de estudiantes que iniciaron el curso de vuelo durante el periodo 2015-2019, la tasa de aprobación es del 73,8 \%, correspondiente a 242 estudiantes. Ahora bien, 86 estudiantes (26,2\%) reprobaron el curso en cualquiera de las dos fases (tabla 5). Precisamente, este porcentaje de reprobación es la cifra por atacar mediante los resultados de la investigación con la implementación de las herramientas didácticas y los métodos de evaluación por parte de personal de pilotos instructores.

\section{Resultados y análisis del sondeo}

El primer resultado obtenido a través del sondeo fue la identificación de tres categorías de análisis, con las cuales se agruparon las herramientas didácticas y los métodos de evaluación inventariados: (1) herramientas didácticas físicas para la clase, (2) descripción de la metodología desarrollada en clase y la implementación de las herramientas, y (3) herramientas de evaluación. 


\section{Tablas de frecuencia del inventario de herramientas didácticas $y$ de evaluación}

Las tablas de frecuencia se elaboraron teniendo en cuenta las categorías de análisis y las variables asociadas a cada categoría: inventario de herramientas didácticas y métodos de evaluación. Con base en una tabulación inicial de la información obtenida de la aplicación de los cuestionarios, se identificaron y seleccionaron las herramientas que se considerarían en la categorización y sistematización de la información.

A partir de la constitución del inventario, se identificaron las siguientes herramientas por categoría con base en las respuestas de los encuestados:

- Herramientas didácticas físicas para la clase: iPad (tableta) y computador por estudiante, videos, PowerPoint, simulador de vuelo, Video Beam, Manual de vuelo, gráficos/poster, maquetas y trabajo de cabina, reportes FAAC-NTSB-UAEAC, elementos de representación gráfica " que subyacen en el empleo de las imágenes con fines didácticos” (Aguirre, 2001, p. 16).

- Descripción de la metodología desarrollada en clase y la implementación de las herramientas: explicación por cada sistema, realimentación por medio de explicación a sus compañeros, explicación basada en ejemplos, clases participativas.

- Herramientas de evaluación: quiz, talleres, exposiciones y pruebas orales.

- Tras la constitución del inventario, se realizó un análisis de frecuencia por herramienta sobre el total de los encuestados, y se estableció un análisis porcentual con el parámetro de uso y no uso, para cada una de ellas (tabla 6). 
Tabla 6. Tabla de frecuencia de herramientas didácticas físicas usadas en clase

\begin{tabular}{lc}
\hline \multicolumn{1}{c}{ Herramienta } & Totales \\
\hline iPad (tableta) y computador por estudiante & 9 \\
Videos & 8 \\
PowerPoint & 11 \\
Simulador de vuelo & 8 \\
Video Beam & 10 \\
Manual de vuelo & 11 \\
Gráficos/poster & 6 \\
Maquetas y trabajo de cabina & 9 \\
Reportes FAA - NTSB - UAEAC & 8 \\
\hline
\end{tabular}

Fuente: elaboración propia.

Con base en el análisis de frecuencias se establecido el análisis porcentual de uso y no uso, sobre el total de los quince instructores encuestados. Luego se estableció un valor de referencia porcentual para definir las herramientas que serían parte de la caracterización a partir de las entrevistas semiestructuradas. De esta forma se estableció como valor de referencia el 46,7 \% como parámetro de selección. Los datos ponderados se presentan en la tabla 7.

Tabla 7. Herramientas didácticas físicas usadas en clase. Porcentaje de uso o no uso

\begin{tabular}{lccc}
\hline Sobre 15 instructores & PowerPoint & Manual de vuelo & Gráficos/posters \\
\hline Porcentaje de uso & $73,3 \%$ & $73,3 \%$ & $40,0 \%$ \\
Porcentaje de no uso & $26,7 \%$ & $26,7 \%$ & $60,0 \%$ \\
\hline Total & $100 \%$ & $100 \%$ & $100 \%$ \\
\hline
\end{tabular}

Fuente: elaboración propia.

La tabla 7 permite evidenciar que la mayoría de los instructores declararon utilizar las herramientas PowerPoint y Manual de vuelo para desarrollar sus clases, ambas con el 73,3\% de uso. Asimismo, se puede afirmar que los datos extremos para esta categoría de investigación corresponden a las variables PowerPoint y Manual de vuelo. Los datos permiten afirmar, además, que la herramienta que menos usan los instructores son los posters, aun sabiendo que 
es un método que visualmente da al estudiante puntos claros para entender los componentes más precisos de la aeronave.

Tabla 8. Metodología en clase por porcentaje de uso o no uso

\begin{tabular}{lccc}
\hline Sobre 15 instructores & $\begin{array}{c}\text { Explicación por } \\
\text { cada sistema }\end{array}$ & $\begin{array}{c}\text { Se basa en } \\
\text { el ejemplo }\end{array}$ & $\begin{array}{c}\text { Clases } \\
\text { participativas }\end{array}$ \\
\hline Porcentaje de uso & $40,0 \%$ & $40,0 \%$ & $73,3 \%$ \\
Porcentaje de no uso & $60,0 \%$ & $60,0 \%$ & $26,7 \%$ \\
Porcentaje de diferencia & $-20,0 \%$ & $-20,0 \%$ & $46,7 \%$ \\
\hline
\end{tabular}

Fuente: elaboración propia.

El análisis porcentual indicó que las clases participativas eran utilizadas por la mayoría de los instructores, con el $73,3 \%$ de uso, mientras que las metodologías de explicación por cada sistema, mediante ejemplos, arrojaron el mayor porcentaje de no uso, con el $60 \%$ (tabla 8). Esto evidencia que los instructores, aunque no utilizan la explicación por cada sistema ni basan su instrucción en el ejemplo, sí utilizan de manera constante la metodología de clases participativas para desarrollar su labor docente. Este indicador permite inferir que el desarrollo de las clases de los docentes se debe orientar de manera más directa a la explicación de cada sistema con el fin de que el estudiante pueda entender más fácilmente el contexto y retomar situaciones locales o mundiales que ayuden a vivenciar a través de la experiencia situaciones de la aviación mundial.

Tabla 9. Herramientas de evaluación por porcentaje de uso y no uso

\begin{tabular}{lcc}
\hline \multicolumn{1}{c}{ Sobre 15 instructores } & Exposición & Pruebas orales \\
\hline Porcentaje de uso & $40,0 \%$ & $73,3 \%$ \\
Porcentaje de no uso & $60,0 \%$ & $26,7 \%$ \\
Porcentaje de diferencia & $20,0 \%$ & $46,7 \%$ \\
\hline
\end{tabular}

Fuente: elaboración propia.

Dentro de la categoría de análisis sobre las herramientas de evaluación el parámetro más alto de uso lo reportó la aplicación de pruebas orales, con el 73,3 \% y el de mayor porcentaje de no uso la exposición, con el 60 \% (tabla 
9), lo cual indica que los instructores prefieren utilizar el método de evaluación por pruebas orales por encima del método de la exposición.

Con base en estos análisis, especialmente la selección por porcentaje de uso y no uso de las herramientas didácticas y de evaluación, se decidió complementar esta información con una selección poblacional de los instructores respecto a la tasa de aprobación o reprobación de los estudiantes formados en sus cursos para aplicar las entrevistas semiestructuradas. Esto se hizo con el fin de describir las herramientas y tratar de obtener información sobre la posible relación entre su uso y la tasa de aprobación y reprobación de los estudiantes.

Tabla 10. Tasa de aprobación por instructores

\begin{tabular}{cccc}
\hline Caso & Estudiantes aprobados & Total de estudiantes & Tasa de aprobación \\
\hline 1 & 15 & 17 & $88 \%$ \\
4 & 14 & 16 & $88 \%$ \\
7 & 25 & 30 & $83 \%$ \\
2 & 21 & 27 & $78 \%$ \\
10 & 20 & 26 & $77 \%$ \\
\hline
\end{tabular}

Fuente: elaboración propia.

En la tabla 10 se observa que la tasa de aprobación en los casos más altos está situada entre el $88 \%$ y el $77 \%$, obtenida del análisis del número total de estudiantes en relación con el número de estudiantes aprobados, e identificando el instructor a cargo del curso en el que se formó a dicho estudiante. Con base en esta tabla se determinó la categorización poblacional de los casos de estudio, ya que concluyó por medio de los datos que estos cinco casos poseen la tasa de aprobación más alta referenciada en el estudio, es decir, que estos instructores son los que han aprobado a más estudiantes, con un total de 95.

Tabla 11. Tasa de reprobación por instructores

\begin{tabular}{cccc}
\hline Caso & Estudiantes reprobados & Total de estudiantes & Tasa de reprobación \\
\hline 15 & 4 & 9 & $44 \%$ \\
13 & 5 & 13 & $38 \%$ \\
14 & 5 & 15 & $33 \%$ \\
9 & 10 & 30 & $33 \%$ \\
3 & 15 & 50 & $30 \%$ \\
\hline
\end{tabular}

Fuente: elaboración propia. 
La tasa de reprobación en los casos más altos está situada entre el $44 \%$ y el $30 \%$, la cual se obtuvo al analizar el número total de estudiantes en relación con el número de estudiantes reprobados e identificando el instructor a cargo del curso en el que se formó a dicho estudiante. Mediante estos resultados cuantitativos podemos determinar de manera parcial que, de acuerdo con la aplicación de los medios didácticos, los casos de reprobación coinciden con los instructores que no utilizaban los medios didácticos para el desarrollo de la clase (tabla 11).

Precisamente, esta población con la mayor tasa de reprobaciones se tomó como referencia para realizar las entrevistas semiestructuradas con las que se continúa ahondando la investigación.

\section{Resultados del análisis de los datos obtenidos en las entrevistas}

A través de las entrevistas al subgrupo poblacional de instructores con mayor tasa de aprobación y reprobación, se obtuvo información detallada sobre el uso de las herramientas didácticas y de evaluación, así como del contexto de utilización.

Las preguntas del cuestionario de entrevistas se agruparon por categoría de análisis y se discriminaron las herramientas didácticas y de evaluación que los pilotos-instructores declararon como de mayor uso y de menor uso. Luego de la aplicación de la entrevista, se realizó la transcripción de las grabaciones de audio y se analizó la información recolectada mediante la codificación de las transcripciones por cada una de las herramientas y por categoría de análisis. Los extractos de las entrevistas se sistematizaron en una matriz de análisis, con referencia a los lineamientos seguidos para este tipo de entrevistas en la educación superior (UNESCO, 2009).

A continuación, se presentan únicamente los resultados del análisis categorial de las entrevistas con el fin de aportar un parámetro de contrastación entre los resultados cuantitativos y el resultado cualitativo de la investigación.

\section{Análisis categorial de las entrevistas para la categoría herramientas didácticas}

Este análisis evidencia que los casos de instructores entrevistados coinciden de manera directa en la utilización en su mayoría del Manual de vuelo 
y del PowerPoint, además determina que en realidad no dan uso a la herramienta de poster, ya que consideran que pueden encontrar más fácilmente esta información en internet. Asimismo, este análisis categorial muestra una estandarización en la forma como desarrollan las clases con un orden lógico adecuado para tal fin.

En consecuencia, se puede afirmar que es necesario incentivar el uso de la herramienta didáctica "bazar", en la cual los estudiantes rotan por varios puntos en donde están diferentes instructores que enseñan el tema y sus derivaciones por medio de maquetas, miniaturas, posters y demás ayudas didácticas que soportan la información adicional con ejemplos cotidianos de situaciones aeronáuticas locales o globales. Lo anterior con el fin de cimentar los conocimientos de los estudiantes. Asimismo, se evidencia que los instructores son conscientes de la importancia de llevar a cabo clases participativas para motivar a cada estudiante.

\section{Análisis de las entrevistas para la categoría herramientas de evaluación}

La verificación de los resultados de este análisis permite plantear el hecho de que los instructores de vuelo por lo general no utilizan la exposición como medio de evaluación para sus clases, sino que utilizan las pruebas orales como parámetro para medir el desempeño de los alumnos.

\section{Discusión}

Con base en las teorías didácticas y los métodos evaluativos que soportan el corpus documental, los resultados obtenidos en este trabajo coinciden con investigaciones anteriores de diferentes campos del conocimiento, en las cuales se evidencia que el uso y la aplicación por parte de los docentes de las teorías de didáctica y de evaluación planteadas en el marco teórico da como resultado un proceso de cognición más efectivo entre el personal de estudiantes en el nivel de educación superior (UNEsCO, 1998), que en este estudio de caso están representados por los oficiales que hacen el curso de Transición de Vuelo.

Durante las cuatro fases de desarrollo, la investigación coordinó de manera eficaz los medios y métodos disponibles. Sin embargo, se suscitaron diferentes obstáculos que podrían suponer un punto de reflexión ante investigaciones posteriores, referentes a la recopilación de datos que no son de común 
estudio dentro del Ejército Nacional, ya que son de carácter reservado y son almacenados de forma clasificada por los líderes militares al mando.

El estudio limitó su alcance a los pilotos instructores de vuelo del BAAVI, que constituyen una muestra representativa de los docentes y estudiantes militares del Ejército Nacional. Esta caracterización se puede definir como un punto de partida para una investigación con un espectro más global que busque incidir de manera más puntual sobre el tema propuesto por el autor.

El aporte teórico de la investigación logró sintetizar las teorías y resultados sobre el aprendizaje significativo, especialmente el presentado por Ausubel y Knowles, referentes al desarrollo del aprendizaje andragógico del ser, la implementación de conceptos de uso de las herramientas didácticas y métodos de evaluación mediante el análisis de la población referentes a sus prácticas de enseñanza y formación en el aula (Valdez, 2017).

En el contexto militar, el aporte se orienta a proponer el desarrollo de una política educativa en el interior del Ejército Nacional con un modelo educativo propio, que garantice el desarrollo efectivo de los procesos de todos los cursos de orden militar adjuntos a la transformación de la doctrina educativa institucional. Así, en palabras de Yáñez (2013), que refuerzan esta afirmación:

contar con un modelo educativo adecuado a su realidad, que establezca la forma en que llevarán a cabo las diferentes tareas encomendadas por la institución y que explicite los principales aspectos que constituyen el marco regulatorio de su quehacer, permite que cada uno de sus integrantes conozca el sentido y dimensión que tiene la actividad que realiza, identifique su rol y sus funciones específicas, así como los deberes y derechos que le asisten; elementos que facilitan el proceso de toma de decisiones en pos de la mejora y contribuyen a la calidad de los procesos implementados en el ámbito educativo, de la investigación y de apoyo a la elaboración y actualización de la doctrina operacional. (p. 54)

La hipótesis planteada en esta investigación fue comprobada: el docente militar no aplica adecuadamente la didáctica ni los métodos de evaluación dentro de su proceso de enseñanza. Como consecuencia, la cognición del estudiante no alcanza los resultados esperados por la institución militar. Pero ¿cuál es el alcance de responsabilidad de la institución en la capacitación efectiva del oficial para el desarrollo de su labor docente? ¿Hasta dónde llega la negligencia de aprendizaje por parte del profesor militar? Estas preguntas se plan- 
tean porque, "conceptualmente, el perfeccionamiento docente incluye tanto la formación inicial de profesores como la actualización profesional" (Donoso, 2008, p. 8). Estos elementos pueden constituirse en un problema de interés institucional susceptible de ser investigado con un propósito plausible de carácter institucional.

\section{Conclusión}

En el proceso de enseñanza de la educación y la doctrina militar, el uso de los componentes didácticos y los métodos de evaluación que implementan los oficiales del Ejército Nacional de Colombia es fundamental para el desempeño de su rol como profesores militares y, por ende, en la cognición efectiva del estudiante.

La falta de aplicación y conocimiento de los componentes didácticos y los métodos de evaluación por parte de los docentes militares en la enseñanza crea fallas en el proceso de cognición de los estudiantes, las cuales derivarán en la aplicación incorrecta de los conceptos cuando pasan de la teoría a la práctica y, posiblemente, en la afectación de las operaciones militares.

\section{Referencias}

Aguirre, M. (2001). Enseñar con textos e imágenes: una de las aportaciones de Juan Amós Comenio. REDIE: Revista Electrónica de Investigación Educativa, 3(1), 1-21. https:// www.redalyc.org/articulo.oa? id=15503101

Alonso, P. (2012). Andragogy as a knowledge driving discipline in higher education. Revista Electrónica Educare, 16(1), 15-26. https://www.redalyc.org/articulo.oa?id=194124281003

Álzate, M. (2015). Aprendizaje significativo por David Ausubel. Revista Educación, (9).

Andrade, A. (2011). Comenio: aportes pedagógicos [Tesis de pregrado, Universidad Pedagógica Nacional]. Repositorio institucional UpN. http://200.23.113.51/pdf/27884.pdf

Chaves, A. (2011). Implicaciones educativas de la teoría sociocultural de Vygotsky. Revista Educación, 25(2), 59-65. https://doi.org/10.15517/revedu.v25i2.3581

Díaz, A. (1997). Didáctica y currículum. Editorial Paidós.

Díaz, A. (1998). La investigación en el campo de la didáctica. Modelos históricos. Perfiles Educativos, (80), 1-23. http://www.redalyc.org/articulo.oa?id=13208002

Donoso, S. (2008). Teacher training in Chile (1990-2007): State versus market? Revista Brasileira de Educação, 13(39), 437-454. https://doi.org/10.1590/s1413-24782008000300003 
Duque, M., \& Packer, M. (2014). Pensamiento y lenguaje. El proyecto de Vygotsky para resolver la crisis de la psicología. Tesis Psicológica: Revista de la Facultad de Psicología, 9(2), 30-57. http://www.redalyc.org/articulo.oa?id=139039784004

Ejército Nacional de Colombia. (2012a). Manual de empleo táctico del Batallón de Aviones EJC 3-4. Imprenta y Publicaciones EJC.

Ejército Nacional de Colombia. (2012b). Manual de misiones tácticas de Aviación EJC 3-175-1. Imprenta y Publicaciones EJC.

Ejército Nacional de Colombia. (2013). Reglamento de vuelo para la Aviación del Ejército EJC 3-176-1. Imprenta y Publicaciones EJC.

Ejército Nacional de Colombia. (2015). Normas para la selección de los aspirantes a cursos en las diferentes especialidades de la Aviación DP 0153. Imprenta y Publicaciones EJC.

Ejército Nacional de Colombia. (2017a). Normas de procedimientos y seguridad operacional para la División de Aviación Asalto Aéreo DP 00217. Imprenta y Publicaciones EJC.

Ejército Nacional de Colombia. (2017b). Programa de entrenamiento para la Aviación del Ejército Nacional DP 00204. Imprenta y Publicaciones EJC.

Hernández, R., Fernández, C., \& Baptista, P. (2014). Metodología de la investigación. McGraw-Hill Interamericana. http://observatorio.epacartagena.gov.co/wp-content/ uploads/2017/08/metodologia-de-la-investigacion-sexta-edicion.compressed.pdf

López, H. (2005). La evaluación como componente del proceso enseñanza-aprendizaje. EduSol, 5(13), 38-46. http://www.redalyc.org/articulo.oa?id=475748652004

Mena, Á., Gutiérrez, E., Flores, O., Gutiérrez, J., Castillejos, D., \& Balcázar, J. (2014). Buenas prácticas respecto a la capacitación docente en la Universidad del Sur 2013... Una mirada a la andragogía. Atenas Revista Cientifico Pedagógica, 4(28), 13-22.

Méndez, A. (2013). Una mirada critica a la educación en el Ejército [Tesis de especialización, Universidad Militar Nueva Granada]. Repositorio institucional. https://repository.unimilitar.edu.co/bitstream/handle/10654/9884/MendezCortesAlvaro2013.pd?sequence=2

Ministerio de Defensa Nacional. (2008a). PEFA. Proyecto Educativo de las Fuerzas Armadas. Imprenta y Publicaciones Fuerzas Militares.

Ministerio de Defensa Nacional. (2008b). PESE Plan Estratégico del Sistema Educativo de las Fuerzas Armadas 2007-2019. Imprenta y Publicaciones Fuerzas Militares.

Ministerio de Defensa Nacional. (2010). SEFA. Lineamientos Curriculares Fuerzas Militares. Imprenta y Publicaciones Fuerzas Militares.

Pérez, D. (2014). Revisión y análisis del modelo de evaluación orientada en los objetivos (Ralph Tyler -1950). https://www.researchgate.net/publication/220025586_Revision_y_ analisis_del_Modelo_de_Evaluacion_Orientada_en_los_Objetivos_Ralph_Tyler_-_1950

Picco, S., \& Orienti, N. (Comp.). (2017). Didáctica y curriculum. Aportes teóricos y prácticos para pensar e intervenir en las prácticas de la enseñanza. Editorial de la Universidad Nacional de La Plata. http://sedici.unlp.edu.ar/handle/10915/61533

Rodríguez, W. (1999). El legado de Vygotsky y de Piaget a la educación. Revista Latinoamericana de Psicología, 31(3), 477-489. https://www.redalyc.org/articulo.oa?id=80531304 
Sotelo, A., \& Arvelo, M. (2016). Proceso participativo para la evaluación y mejora del desempeño estudiantil. Revista Lusófona de Educação, (33), 143-159. https://www.redalyc. org/articulo.oa?id=34949131011

Terreros, O. (2012). Investigando un modelo pedagógico desde y para lo castrense. TecnoESUFA, (18), 8-16. https://publicacionesfac.com/index.php/TecnoESUFA/article/view/373/493

Unesco. (1998). Declaración mundial sobre la educación superior en el siglo XXI: visión y acción. Educación Superior y Sociedad, 9(2), 97-113. http://www.iesalc.unesco.org/ess/ index.php/ess3/article/view/171/162

Unesco. (2009). Conferencia mundial sobre la educación superior - 2009: la nueva dinámica de la educación superior y la investigación para el cambio social y el desarrollo. (Sede de la Unesco, París, 5-8 de julio de 2009). Revista da Avaliação da Educação Superior (Campinas), 14(3), 755-766. https://doi.org/10.1590/s1414-40772009000300013

Unidad Administrativa Especial de Aeronáutica Civil [UAEAC]. (2020). Reglamentos Aeronáuticos de Colombia. RAC 141. https://www.aerocivil.gov.co/normatividad/ RAC/RAC\%20\%20141\%20-\%20Centros\%20de\%20Instrucci\%C3\%B3n\%20aeron\%C3\%A1utica\%20 civil.pdf

Valdez, G. (2017). El modelo pedagógico de Juan Amós Comenio y el desarrollo de la revolución económica del siglo XVII. http://www.academia.edu/10976343/El_ modelo_pedagógico_de_Juan_Amós_Comenio_y_el_desarrolla_de_la_revolución_ económica_del_siglo_XVII

Yáñez, L. (2013). El modelo educativo como herramienta eficaz para impulsar los cambios derivados del proceso de modernización de la educación militar. Revista de Educación del Ejército de Chile, (40), 47-58. http://www.seade.cl/paginas/not/revistaEducacion2013.pdf 